\title{
WAYS TO IMPROVE NATURAL FERTILITY
}

\author{
Tetiana V. Fartushok ${ }^{1}$, Halyna B. Semenyna' , Oksana M. Yurchyshyn² ${ }^{2}$ Olha S. Komissarova ${ }^{3}$ \\ 'DANYLO HALYTSKY LVIV NATIONAL MEDICAL UNIVERSITY, LVIV, UKRAINE \\ ${ }^{2}$ IVAN HORBACHEVSKY TERNOPIL NATIONAL MEDICAL UNIVERSITY, TERNOPIL, UKRAINE \\ ${ }^{3}$ SHUPYK NATIONAL HEALTHCARE UNIVERSITY OF UKRAINE, KYIV, UKRAINE
}

\begin{abstract}
The aim: Based on the study of the results of retrospective analysis, optimize ways to improve natural fertility.

Materials and methods: Data from the American Society for Reproductive Medicine and the Society for Reproductive Endocrinology and Infertility were used to study this problem. Conclusions: With a body mass index $>35$, the time reguired for conception increases by 2 times. At the same time, when the body mass index $<19$, the time reguired for conception increases by 4 times. Increased levels of mercury in seafood are associated with infertility. Smoking accelerates the rate of exhaustion of the ovarian follicular apparatus. When trying to become pregnant, it is advisable to avoid drinking more than 2 servings per day. Caffeine intake over 5 cups/day in women correlates with reduced fertility.
\end{abstract}

KEY WORDS: fertility, reproductive capacity, infertility, cervical mucus

Wiad Lek. 2021;74(1):144-149

\section{INTRODUCTION}

In their daily practice, doctors often encounter patients' requests for advice on optimizing conditions for the implementation of their reproductive function. However, to date, there has not been developed single evidence-based clinical guidelines that would clearly regulate this issue. Therefore, at the beginning of 2017, the American Society for Reproductive Medicine (American Society for Reproductive Medicine) and the Society for Reproductive Endocrinology and Infertility (Society for Reproductive Endocrinology and Infertility) published their findings on ways to increase the incidence of pregnancy in family couples / individuals in the absence of of them proven reasons for infertility.

\section{THE AIM}

Based on retrospective analysis, optimize ways to improve natural fertility.

\section{MATERIALS AND METHODS}

Data from the American Society for Reproductive Medicine and the Society for Reproductive Endocrinology and Infertility were used to investigate this problem. The work envisages measures to enure human health, human dignity and moral and ethical standards in accordance with the principles of the Helsinki Declaration of Human Rights, the Council of Europe Convention on Human Rights and Biomedicine and relevant laws of Ukraine (opinion of Danylo Halytsky Lviv National Medical University Bioethics Commission).

\section{REVIEW AND DISCUSSION}

\section{FERTILITY AND AGE}

Fertility is defined as the ability to procreate. With the probability of conception remaining relatively constant from cycle to cycle in some individuals, in general in the population it is usually the highest during the first months of regular sexual life without the use of contraceptives or the entry of sperm to the female genital tract, then gradually decreases. About $80 \%$ of couples manage to conceive a baby in the first 6 months since the beginning of the attempts. The monthly fertility rate is highest in the first 3 months. Fertility is reduced by about half of the women below the age of 40 compared with persons aged 20 years and older.

Fertility varies among different groups of people and decreases with age in both men and women, but the effect of aging is much more pronounced among females. In women, the chances of getting pregnant are significantly reduced after 35 years. Although men also after age 35 begin to deteriorate spermogram parameters, their fertility is not disturbed to about 50 years.

Infertility is defined as the inability of couples in the reproductive age to conceive a child during regular sexual life without the use of contraceptives throughout the year. The appointment of an examination and treatment at an earlier date may be justified by individuals on the basis of their anamnesis and shown to women who are 35 years of age and over after 6 months of unsuccessful attempts to become pregnant in view of the age-related decline in fertility. 


\section{FREQUENCY OF SEXUAL ACTS}

Over the past decade, a certain amount of literary data has been accumulated concerning the optimal frequency of sexual relations. While sexual restraint over 5 days may adversely affect sperm count, similar intervals of up to 2 days are associated with their normal density. The false idea is that frequent ejaculation reduces malnutrition. E. Levitas et al. (2015) in a retrospective study, analyzing about 10,000 samples of semen, found that the concentration of sperm and their mobility remained normal even with daily ejaculation.[1] The researchers also found that the above-mentioned indicators for men with oligozoospermia may be the highest in daily ejaculation. Non-spontaneous sexual restraint does not mainly affect the morphology of sperm, but if it lasts more than 10 days, then the spermogram parameters begin to deteriorate. Despite the fact that the definition of indicators of spermogram gives valuable quantitative data, with their help can not make an exact conclusion about the fertility of sperm.

Although evidence suggests that daily sexual intercourse has little benefit in terms of successful fertilization, the need to exercise them on certain days or even at certain times of the day can cause severe stress. Stress associated with attempts to conceive can adversely affect the quality of sexual life, reduce sexual desire, lead to a decrease in the frequency of sexual acts. These events can become even more acute when coitus schedules are scheduled based on a predicted ovulation time [2]. Marital couples should be informed that reproductive capacity is increasing with increasing frequency of sexual acts and is the highest if they occur every 1-2 days. It should be noted that the optimal frequency of sexual relations is determined according to their own preferences.

\section{FERTILITY WINDOW}

«Fetal window» or «Fertility window» is a period of time that includes five days before and on the day of ovulation, they denote a 6-day interval that ends on the day of ovulation. Theoretically, the viability of both oocytes and sperm is maximal during this time. From the clinical point of view, the maximum fertility interval can be determined by analyzing the duration of the menstrual cycle (MC), the results of ovulation tests and the characteristics of the mucus of the cervical canal.

A sexually transmitted infection with the highest probability may end with fertilization if it occurs within a 3-day interval ending on the day of ovulation. In a study by A.J. Wilcox et al. (1995) with the participation of 221 fertile women, fertility was maximal if sexual intercourse was preceded by ovulation (in 2 days) (Fig. 1).[3] Another study (Dunson D. B. et al., 2010), devoted to family planning, analyzed the results of two cohorts of the subjects surveyed [4]. In one, basal temperature was measured to determine the ovulation time, while in the other, the content of estrogen / progesterone metabolites in the urine was measured. Probability of pregnancy was the highest if sexual intercourse occurred the day before ovulation, and began to decrease on the foreseeable day of its onset.
Among women who described their $\mathrm{MC}$ as generally regular, the likelihood of conception as a result of a one-time sexual intercourse increased during a prolonged period [5]. The probability of clinical pregnancy increased from $3.2 \%$ on the 8 th day of the cycle to $9.4 \%$ on the 12 th and decreased to $<2 \%$ on the 21 st day of MC. It also shows a reduction in the chances of conception when increasing the age of women (Fig. 2). However, the ability to fertilize increases with an increase in the frequency of sexual intercourse during the fertility window [6]. As it is unpredictable to predict the day of ovulation, it is difficult to use any of the available methods for determining it, family pairs should be encouraged to intensify their sexual life shortly after menstruation and up to ovulation (provided that the $\mathrm{MC}$ is regular in women). Due to the fact that the duration of the productive window in women may be different, the probability of conception in them also varies [7]. That is why regular sexual activity is the best advice to increase the chance of a pregnancy.

\section{DEFINITION OF OVULATION}

The peak of the fertile period can change significantly even in women with regular MCs. Those who monitor their cycle and monitor changes in cervical mucus, libido, pain and mood can accurately predict the time to ovulate only in half of the cases. Although there is no evidence that the definition of ovulation increases the ability to conceive, the time of sexual intercourse is important and, therefore, should be determined by various methods of predicting ovulation.

A study of cervical mucus is a simple and effective way to determine ovulation (Fig. 3). The estimated probability of conception is the highest, if the mucus is delicate and transparent, but the detection of such secretions is not a prerequisite for the onset of pregnancy. The amount of cervical mucus increases with an increase in the concentration of estrogens in the plasma during 5-6 days preceding ovulation, and reaches its peak within 2-3 days after it. D. B. Dunson et al. (2011) in a prospective study, which included an estimate of $2832 \mathrm{MCs}$, found that changes in cervical mucus are closely correlated with basal temperature, and with this method, it is possible to predict the peak of the fertile period more precisely than during the menstrual calendar.[8].

Ovulation tests, including luteinizing hormone (LH) levels in urine, are widely used to determine the period of maximum probability of conception. Although numerous studies have confirmed the accuracy of methods for determining the peak of $\mathrm{LH}$ in urine in the middle of $\mathrm{MC}$, ovulation can occur at any time during the next 2 days, and false-positive results occur in approximately $7 \%$ of cycles. Although the monitoring of urinary LH levels can help reduce the time interval before conception in pairs with infrequent sexual intercourse, in a large study by J. L. Bigelow et al. (2014) found that changes in cervical mucus during MC can predict the days of the highest probability of fertilization with the same or even greater accuracy than 

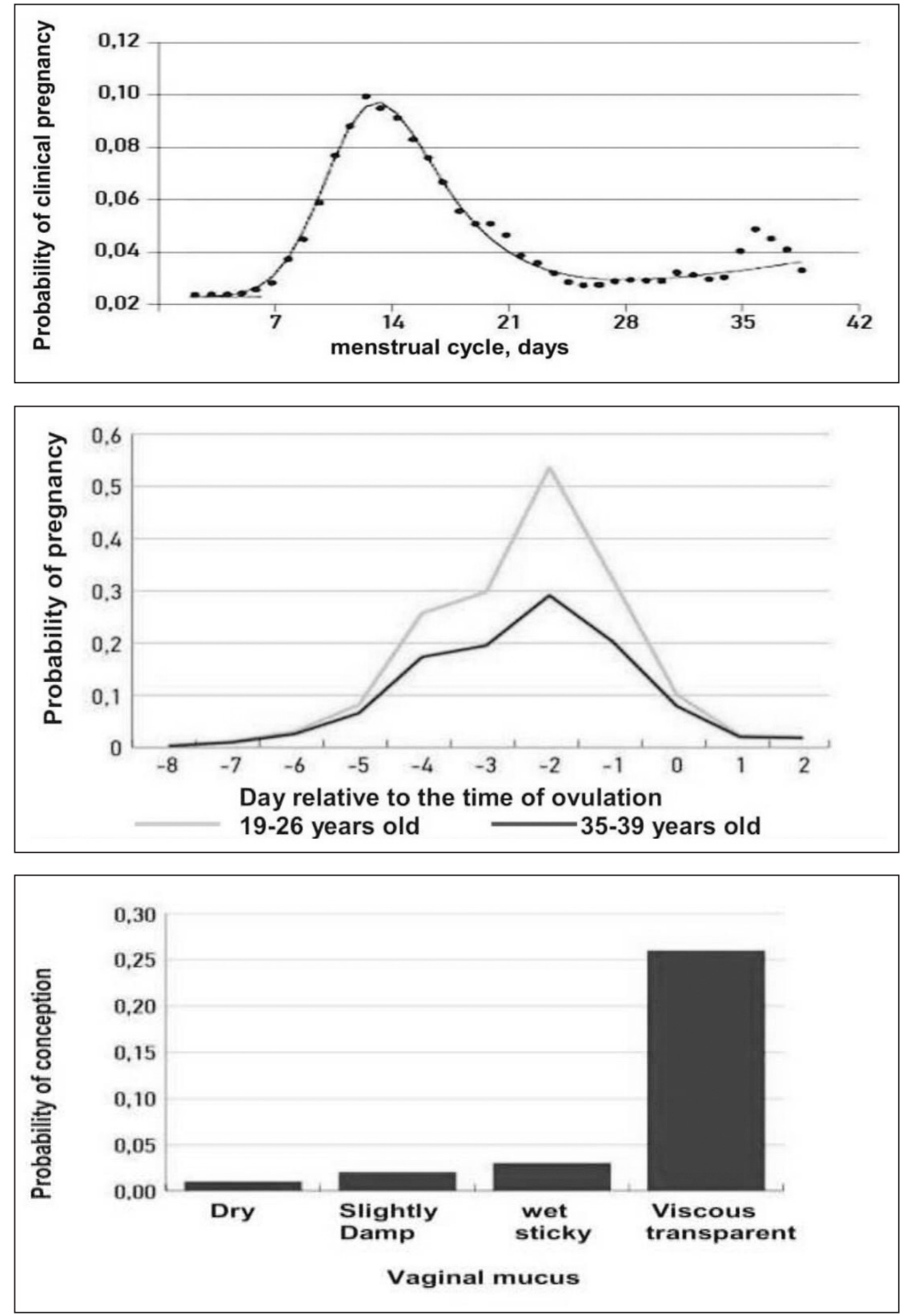

Fig. 1. Probability of the onset of pregnancy depending on the day of the menstrual cycle with a one-time sexual intercourse. Adapted for Dunson et al. (1999).

Fig.2. Probability of the onset of pregnancy depending on the day of the menstrual cycle, including repeated sexual intercourse, in patients of all ages. Adapted for J. B. Stanford, D.B. Dunson (2007).

Fig.3. Estimation of the probability of conception on the characteristics of vaginal secretion on the day of sexual intercourse. Adapted for Scarpaet al. (2006). when measuring basal temperature and monitoring the level of LH in urine.[9].

\section{FEATURES OF THE SEXUAL LIFE \\ OF THE MARRIED COUPLE}

Many women believe that staying in a lying position after sexual intercourse facilitates the transport of sperm and prevents sperm out of the vagina. However, this representation does not have any scientific basis.

Spermatozoids can be identified in fallopian tubes after 15 minutes after reaching the cervix in the middle of the MC. After that, they pass through the fallopian tube to the abdominal cavity.

There is not enough evidence to suggest that fertility has an effect on the ability to fertilize during sexual intercourse. It is known that sperm can be detected in the cervical canal a few seconds after ejaculation, regardless of the cohort position. Although female orgasm may contribute to the transport of sperm, correlations between orgasm and fertility are not established. There is also no conclusive evidence of the existence of a link between the characteristics of the sexual behavior of the couple and the child's article.

According to the results of studies on the effects of lubricants on the survival of sperm in vitro, it was concluded that the use of some of them may reduce fertility. While available on sale water-based lubricants inhibit sperm motility in vitro from 60 to $100 \%$ for 60 minutes of incubation, canola oil (rape) does not have a similar harmful effect [10]. Unlike mineral oil, a water-based gel-lubricant with no special additives (KY Jelly), olive oil and saliva, diluted to a concentration of $<6.25 \%$, negatively affects the mobility and speed of spermatozoa [11]. Lubricants based on hydroxyethyl cellulose also have no noticeable negative 
Table I. Life style factors that affect fertility

\begin{tabular}{cc}
\hline Factor & Effect on Fertility \\
\hline Obesity (body mass index $>35)$ & The time required for conception increases by 2 times \\
\hline Body mass deficiency (body mass index <19) & The time required for conception increases 4 times \\
\hline Smoking & The relative risk of infertility increases by $60 \%$. \\
\hline Drinking $(>2$ servings of drinks / day)* & The relative risk of infertility increases by $60 \%$. \\
\hline Caffeine & Ability to fertilize is reduced by $45 \%$. \\
\hline Narcotic substances & Relative risk of infertility increases by $70 \%$. \\
\hline Toxins, solvents 1 portion of alcoholic beverage $=10-12 \mathrm{~g}$ of alcohol; This is 1 glass of vodka or cognac $(25-30$ ml) or $1 \mathrm{glass}$ of wine $(100-120 \mathrm{ml})$, \\
or 1 small beer mug $(220-260 \mathrm{ml})$.
\end{tabular}

effect on spermogram parameters [12]. Despite the above results of studies on the negative effects of some in vitro sperm liquids, their use in couples trying to conceive a child does not interfere with fertilization [13].

Thus, recommended for use in case of need are lubricants based on mineral oil, canola oil or hydroxyethyl cellulose.

\section{DIET AND LIFESTYLE}

It is known that fertility among women, both overweight and underweight, is reduced. At the same time, data on the correlation of body weight and fertility in ovulating women is very small (Table 1). Increased levels of mercury in the blood due to the consumption of seafood containing heavy metals is associated with infertility $[14,15]$. Women planning pregnancy should be advised to take folic acid supplements (a minimum daily dose of 400 micrograms) to reduce the risk of developing neural tube defects [16].

\section{SMOKING}

Smoking has been shown to have a negative effect on fertility. In the course of the large meta-analysis (Augood C. et al., 2018), when comparing the health status of 10,928 women smokers with 19,128 non-smokers, it has been found that in the presence of this harmful habit, infertility is much more frequent (odds ratio [ VH] 1.60; 95\% confidence interval [DII]: 1.34-1.91) [17]. According to research findings menopause occurs on average 1-4 years earlier in female smokers than in non-smokers.[18] This indicates that smoking accelerates the rate of depletion of the follicular apparatus of the ovaries. Although smokers are diagnosed with reduced sperm count, reduced mobility, and morphology, there is no convincing evidence that smoking reduces malnutrition $[19,20]$.

\section{ALCOHOL CONSUMPTION}

The effect of alcohol on female fertility is not surely studied. Meanwhile, some scientists came to the conclusion that it has a negative effect, while others believe that its use can increase fertility. In a Swedish prospective study involving 7393 women, the risk of infertility was substantially increased (BP 1.59; 95\% CI: 1.09-2.31) among those who consumed daily 2 portions of alcoholic beverages. [20] At the same time, this risk decreased (BP 0.64; 95\% CI: 0.46$0.90)$ for those who drank $<1$ portion of drink per day. Other studies have shown that increased alcohol intake reduces the likelihood of conception [21].

In contrast to the aforementioned data in the Danish study with the participation of 29,844 pregnant women, it was demonstrated that the period before conception was shorter in women who drank wine than those who did not drink alcohol.[22] In a study involving 1769 Italian women in the postpartum period the authors found no link between alcohol intake and conceiving difficulties. [23]

When trying to get pregnant it is advisable to avoid drinking more alcohol ( $>2$ servings of drinks / day). However, there is limited evidence that its more moderate consumption negatively affects fertility. Undoubtedly, in the course of pregnancy, alcoholic beverages should be completely stopped as they have an established adverse effect on the development of the fetus; At the same time, no «safe» level of their consumption is not defined. In men, the use of alcohol does not aggravate spermogram parameters [24].

\section{USE OF CAFFEINE}

Scientists have found that high levels of caffeine intake (500 mg; 5 cups of coffee or its equivalent per day) correlate with reduced fertility (VH 1.45; 95 \% CI: 1.03-2.04) [25]. In general, moderate consumption of caffeine (from 1 to 2 cups of coffee or its equivalent per day) before or during pregnancy does not cause a clear adverse effect on fertility and the effects of pregnancy. In men, caffeine intake does not change spermogram parameters [26].

On the basis of literature review, it was concluded that attending a sauna does not reduce female fertility and is safe during uncomplicated pregnancy [27]. Recommendations for controlling or reducing the testicular heat effects in healthy men are currently not supported [24]

The impact of environmental toxins today is recognized as a potential cause of reduced fertility. Ability to fertilize can be reduced in women who are exposed to certain toxins and solvents, including those used in the dry-cleaning and printing industry. In addition, men who are in contact with heavy metals are more likely to be diagnosed with abnormal 
spermogram parameters [28]. Pesticides can negatively affect the reproductive health of agricultural workers [29].

The impact of environmental toxins today is recognized as a potential cause of reduced fertility. Ability to fertilize can be reduced in women who are exposed to certain toxins and solvents, including those used in the dry-cleaning and printing industry. In addition, men who are in contact with heavy metals are more likely to be diagnosed with abnormal spermogram parameters [28]. Pesticides can negatively affect the reproductive health of agricultural workers [29]. The number of studies on the evaluation of the action of pesticides on the parameters of spermogram is increasing [30]. In addition, studies on animal models have shown that adverse environmental factors can cause serious reproductive impairment [31]. In particular, contact with lead and industrial microwave ovens is likely to be better avoided or minimized $[32,33]$.

\section{CONCLUSIONS}

- The fruitful window covers a 6-day interval that ends on the day of ovulation, and correlates with the number and characteristics of cervical mucus.

- Frequent sexual intercourse (every 1-2 days) during the "window of fertility» is associated with the highest incidence of pregnancy, but the latter are almost equivalent to less active sexual life (2-3 times a week).

- Accepting certain corytic poses, as well as finding a woman lying in a position after sexual intercourse do not have a significant effect on fertility.

- The use of methods for the detection and prediction of ovulation is appropriate for couples with infrequent sexual relations.

- Moderate alcohol consumption (1-2 servings of drinks per day) and caffeine may adversely affect fertility.

- The period of time required for conception increases with age. For women over 35 years after 6 months of unsuccessful attempts to become pregnant, consultation of the reproductionist is indicated.

- In individuals with regular MC sexual intercourse every 1-2 days from the beginning of the period of the fruitful window can maximize the chances of fertilization.

- Couples who try to conceive a child should avoid smoking, excessive alcohol consumption ( $>2$ servings of beverages per day), and use of most of the available vaginal lubricants.

\section{REFERENCES}

1. Levitas E., Lunenfeld E., Weiss N. et al. Relationship between the duration of sexual abstinence and semen quality: analysis of 9,489 semen samples. Fertility and Sterility. 2015;83(6):1680-1686.

2. Tolley E.E., Severy L.J. Integrating Behavioral and Social Science Research Into Microbicide Clinical Trials: Challenges and Opportunities. American Journal of Public Health. 2016; 96(1): 79-83.

3. Wilcox A.J., Weinberg C.R., Baird D.D. Timing of sexual intercourse in relation to ovulation. Effects on the probability of conception, survival of the pregnancy, and sex of the baby. The New England journal of medicine. 2015; 7;333(23):1517-1521.
4. Dunson D.B., Baird D.D., Wilcox A.J., Weinberg C.R. Day-specific probabilities of clinical pregnancy based on two studies with imperfect measures of ovulation. Human Reproduction. 2019;14(7):1835-1839.

5. Wilcox A.J., Baird D.B., Dunson D.B., Mcconnaughey D.R. On the frequency of intercourse around ovulation: Evidence for biological influences. Human Reproduction. 2014;19(7):1539-1543.

6. Stanford J., Dunson D. Effects of sexual intercourse in time to pregnancy studies. American Journal of Epidemiol. 2017;165:1088-1095.

7. Keulers M.J., Hamilton C.J.C.M., Franx A., Ewvers J.L.H. The length of the fertile window is associated with the chance of spontaneously conceiving an ongoing pregnancy in subfertile couples. Human Reproduction. 2017:22:1652-1656.

8. Dunson D.B., Sinai I., Colombo B. The Relationshipbetween Cervical Secretions and the Daily Probabilities of Pregnancy: Effectiveness of the Two Day Algorithm. Human Reproduction. 2017 :16: 2278-2282.

9. Dunson D.B., Bigelow J.L., Colombo B. Reduced fertilization rates in older men when cervical mucus is suboptimal. Obstetrics and Gynecology.2015:105:788-793.

10. KuttehW. H. Antiphospholipid antibody-associated recurrent pregnancy loss: treatment with heparin and low-dose aspirin is superior to lowdose aspirin alone. American Journal of obstetrics and gynecology. 2016;174(5):1584-1589.

11. Anderson L., Lewis S.E., McClure N.The effects of coital lubricants on sperm motility in vitro. Human Reproduction. 2018;13(12):3351-3356.

12. Agarwal A., Deepinder F., Sharma R.K., Ranga G. Effect of cell phone usage on semen analysis in men attending infertility clinic: an observational study. Fertility and Sterility. 2018;89(1):124-8.

13. Steiner A.Z., Long D.L., Tanner C., Herring A.H. Effect of Vaginal Lubricants on Natural Fertility. Obstetrics and Gynrcology. 2012:120 (1):44-51.

14. Choy C.M., Lam C.W., Cheung L.T., Briton-Jones C.M. et al. Infertility, blood mercury concentrations and dietary seafood consumption: a case-control study. BJOG: an international journal of obstetrics and gynecology. 2012;109(10):1121-1125.

15. Yablonskaya S.V., Fartushok T.V., Veselsky S.P., Kondratyuk 0.A. et al. Violation of lipid composition and properties of the plasma membrane of the epithelial cells of the villous chorion of the placenta under the influence of chlamydial infection. Ukr. Biochem. Journal. 2008;80(2):100-105.

16. De-Regil L.M., Fernández-Gaxiola A.C., Dowswell T., Peña-Rosas J.P. Effects and safety of periconceptional folate supplementation for preventing birth defects. The Cochrane Database of systematic rewiews. 2010: 6;(10):CD007950.

17. Augood C., Duckitt K., Templeton A.A. Smoking and female infertility: a systematic review and meta-analysis. Human Reproduction.2018;13(6):1532-1539.

18. Adena M.A, Gallagher H.G.Cigarette smoking and the age at menopause. Annals of human biology. 2012;9(2):121-30.

19. Greenberg E.R, Baron J.A,Stukel T.A et al.A clinical trial of beta carotene to prevent basal-cell and squamous-cell cancers of the skin. The Skin Cancer Prevention Study Group. The New England journal of medicine. 2010:20;323(12):789-95.

20. Povey A.C, Clyma J.A., McNamee R. et al.Modifiable and non-modifiable risk factors for poor semen quality: a case-referent study. Human reproduction. $2012 ; 27(9): 2799-806$.

21. Eggert H., Gortchakov A., Saumweber H. Identification of the Drosophila interband-specific protein $Z 4$ as a DNA-binding zincfinger protein determining chromosomal structure. Journal of Cell Science.2014; 117:4253-4264. 
22. Hassan M.A., Killick S.R. Negative lifestyle is associated with a significant reduction in fecundity.Fertility and sterility. 2014;81(2):384-92.

23. Huhl M., Olsen J., Andersen A-M.N., Gronbaek M. Intake of wine, beer and spirits and waiting time to pregnancy. Human Reproduction. 2013;18 (9):1967-1971.

24. Chiaffarino F., Parazzini F., La Vecchia C., Chatenoud L. et al. Obstetrics and gynecology. $2019 ; 94(3): 395-398$.

25. Povey A.C., Clyma J.A., McNamee R., Moore H.D. et al. Participating Centres of Chaps-uk.Modifiable and non-modifiable risk factors for poor semen quality: a case-referent study. Human Reproduction. 2012 ;27(9):2799-2806.

26. Bolúmar F., Olsen J., Rebagliato M., Bisanti L. Caffeine intake and delayed conception: a European multicenter study on infertility and subfecundity. European Study Group on Infertility Subfecundity. American journal of epidemiology. 2017;145(4):324-334.

27. Bonde J.P, Ernst E., Jensen T.K. et al. Relation between semen quality and fertility: a population-based study of 430 first-pregnancy planners. Lancet. 2018:10;352(9135):1172-1177.

28. Hannuksela M.L., Ellahham S. The Effects of Coffee and Korean Red Ginseng with Body Wrap Steam Bathing on Stress Markers and Lipid Profiles. Journal of Food and Nutrition Research. 2015;3(4):246-251.

29. Hruska K., Slana I., Kralik P., Pavlik I. Mycobacterium avium subsp. paratuberculosis in powdered infant milk: F57 competitive real time PCR. Veterinarni Medicina. 2011;56(5): 226-230.

30. Snijder C.A., Kortenkamp A., SteegersE.A., JaddoeV.W. et al. Intrauterine exposure to mild analgesics during pregnancy and the occurrence of cryptorchidism and hypospadia in the offspring: the Generation R Study. Human Reproduction. 2012;27(4):1191-1201.

31. Kyselova V., Peknisova J., Buskiova D., Boubelik M. Effects of p-nonylphenol and resveratrol on body and organ weight and in vivo fertility of outbred CD-1 mice. Reproductive Biology and Endocrinology. 2013;30:427-435.

32. Skakkebaek N.E, Rajpert-De Meyts E., Main K.M. Testicular dysgenesis syndrome: an increasingly common developmental disorder with environmental aspects. Human Reproduction. 2011;16(5):972-978.

33. Weyandt T.B, Schrader S.M, Turner T.W, Simon S.D. Semen analysis of military personnel associated with military duty assignments. Reproductive Toxicology. 2016;10(6):521-528.
Research work of the department: IMPROVING MONITORING OF OBSTETRIC CARE FOR IDIOPATHIC PREGNANCY MISCARRIAGE. Terms 2017-2022. State registration number 0117 U001080. Sources of funding within the framework of state funing of the research work of the Department of Obstetrics and Gynecology of Danylo Halytsky Lviv National Medical University.

\section{ORCID and contributionship:}

Tetiana V. Fartushok: 0000-0001-6571-0108 ${ }^{A, D}$

Halyna B. Semenyna: 0000-0003-2247-6731 B,F

Oksana M. Yurchyshyn: 0000-0002-0754-2983 ${ }^{\mathrm{C}}$

Olha S. Komissarova: 0000-0001-7513-2217 ${ }^{E}$

\section{Conflict of interest:}

The Authors declare no conflict of interest.

\section{CORRESPONDING AUTHOR Tetiana V. Fartushok}

Danylo Halytsky Lviv National Medical University, 69 Pekarska str., 79010 Lviv, Ukraine tel: +380973363150 e-mail: fartushok1@ukr.net

Received: 09.03 .2020

Accepted: 08.10 .2020

\footnotetext{
A - Work concept and design, B - Data collection and analysis, C - Responsibility for statistical analysis, D-Writing the article, $\mathbf{E}$-Critical review, $\mathbf{F}$ - Final approval of the article
} 